\title{
Effect of Junction Resistance on the Percolation Resistivity of Metal Nanowire Networks
}

\author{
Kevin Thomas DeStefano \\ University of Florida
}

Faculty mentor: Ant Ural, Department of Electrical and Computer Engineering

\begin{abstract}
There has been significant research interest recently in random networks of one-dimensional elements such as nanotubes and nanowires. In particular, metal nanowire networks exhibit high transmittance, low sheet resistance, mechanical flexibility, and fast deposition. These unique properties make metal nanowire networks promising candidates as transparent, conductive electrodes. In this work, we perform Monte Carlo simulations to study the effect of the wire-to-wire junction resistance on the resistivity and percolation critical exponents of metal nanowire networks. We compute the network resistivity as a function of the wire-to-wire junction resistance over six orders of magnitude, ranging all the way from a junction resistance-dominated to a nanowire resistance-dominated network. We study this effect when other nanowire/device parameters are also varied. We also investigate the effect of the wire-to-wire junction resistance on the percolation critical exponents over a wide range of nanowire and device parameters. The junction resistance plays a critical role in determining both the resistivity and the critical exponents of metal nanowire networks. These studies illustrate how the junction resistance affects the macroscopic resistivity of the network. They also show that Monte Carlo simulations are an essential tool for providing insights into the percolation resistivity of transparent, conductive metal nanowire networks.
\end{abstract}

\section{Introduction}

There has been significant research interest recently in thin films consisting of random networks of one-dimensional (1D) nanoelements, such as carbon nanotubes, graphene nanoribbons, and metal nanowires, since they are promising candidates for next-generation transparent conductors, which are used in many device applications such as touch screens, flat panel displays, solar cells, light-emitting diodes (LEDs), and wearable flexible electronics (Hecht et al., 2011; Coleman and De, 2011; Hu et al., 2011; Ye, 2014). In particular, Ag and Cu nanowire networks exhibit high transmittance, low sheet resistance, mechanical flexibility, and solution-based fast deposition (Hecht et al., 2011; Coleman and De, 2011; Hu et al., 2011; Ye, 2014; Langley et al., 2013; Borchert et al., 2015; Ackermann et al., 2016; Aurang et al., 2016). 
These unique properties make metal nanowire networks emerging candidates to replace indium tin oxide (ITO), which suffers from brittleness, scarcity, high cost, and slow deposition.

At the high optical transmittance values required for transparent conductors, metal nanowire networks need to be very thin. At those thicknesses, the electrical transport and resistivity in these networks deviate from their bulk properties and are governed by percolation theory (Stauffer and Aharony, 1985). As a result, Monte Carlo simulations need to be employed to theoretically calculate the percolation resistivity of networks consisting of $1 D$ nanoelements (Jagota and Tansu, 2015; Behnam and Ural, 2007; Simoneau et al., 2015; White et al., 2009; Berhan and Sastry, 2007). However, most simulation work on 1D nanoelement networks has modeled the nanowires as straight sticks. In reality, the nanotubes and nanowires in networks deposited experimentally exhibit some degree of curviness (Ye et al., 2014; Aurang et al., 2016; Kim et al., n.d.; Ye et al., 2013; Azuma et al., 2013). There has been some theoretical work on the effect of curviness on the percolation threshold in carbon nanotube/nanofiber networks (Yi et al., 2004) and nanocomposites (Berhan and Sastry, 2007; Chunyu, 2007; Yu et al., 2013; H.M. et al., 2010), showing that curviness increases the percolation threshold of random networks.

Curviness not only affects the percolation threshold, but also the resistivity of the network, which determines the sheet resistance of transparent conductors. In a few previous studies, it has been shown that waviness decreases the conductivity of carbon nanotube networks (Simoneau et al., 2015) and three-dimensional (3D) carbon nanotube-based nanocomposites (Bao et al., 2013; Takeda et al., 2011; Li et al., 2007; Dalmas et al., 2006). However, a systematic and comprehensive study of the effect of nanowire curviness on the scaling of percolation resistivity in metal nanowire networks and its interaction with other nanowire/device parameters, in particular nanowire alignment, is currently lacking.

In this work, we systematically study the effect of junction resistance on the percolation resistivity of metal nanowire networks by Monte Carlo simulations. We develop a method to generate curvy nanowires using $3^{\text {rd }}$-order Bézier curves and the concept of curviness angle, which puts a geometrical constraint on the statistical distribution of nanowire curviness in the network. We then systematically study the effect of curviness on the network resistivity and its interaction with two other nanowire/device parameters, namely nanowire density and nanowire length 
The results presented in this work are not limited to metal nanowire networks, but can be applied to any junction-resistance dominated two-dimensional (2D) or vertically stacked quasi2D network, film, or nanocomposite consisting of 1D nanoelements such as carbon nanotubes, graphene nanoribbons, nanowires, and nanorods. Our results show that computational studies are an essential tool for developing a better physical understanding of percolation transport and resistivity scaling in transparent, conductive nanowire networks and predicting/optimizing their electronic properties for a wide range of applications.

\section{Computational Method}

\section{Generation of the Network}

We employed Monte Carlo simulations to study the electrical characteristics of curvy nanowire networks. A similar procedure for generating straight nanowires as 1D width less "sticks" and calculating the resistivity of the network has been explained in detail in our previous work (Behnam and Ural, 2007; Behnam et al., 2007; Hicks et al, 2009; Behnam et al., 2008). Briefly, each nanowire in the network is modeled as a stick with a fixed length $l_{W}$ with a random position and direction on a 2D grid defined by the device length $L$ and the device width $W$, lying between two electrodes. Nanowires are first generated in a rectangular area larger than the device area by a length equal to $l_{W}$ on each side; subsequently, only nanowires lying in the inner rectangle defined by $L$ and $W$ are used in the resistivity calculation. This random generation of nanowires on the 2D grid is repeated for five vertically stacked layers to form the network with the desired value of the nanowire density $n$, which is defined as the total number of nanowires in all five layers per unit area in units of $\square \mathrm{m}^{-2}$. It is assumed that only nanowires in the same or nearest neighbor 2D layers form junctions, and the farther away layers are non-interacting. This approach was chosen to more realistically model experimentally studied metal nanowire networks and thin films.

We used $3^{\text {rd }}$-order (cubic) Bézier curves to mathematically generate curvy nanowires. A cubic Bézier curve $B(t)$ is a parametric function given by

$$
B(t)=(1-t)^{3} P_{0}+3(1-t)^{2} t P_{1}+3(1-t) t^{2} P_{2}+t^{3} P_{3}
$$

where the parameter $t$ is defined in the range $0 \leq t \leq 1$, and the function is specified by the values of the four control points, namely $P_{0}, P_{1}, P_{2}$, and $P_{3}$, whose $t$ coordinates are $0,1 / 3,2 / 3$, 
and 1, respectively. By definition, $P_{0}$ is the start and $P_{3}$ is the end point of the curve. As a result, the degree of curviness of the nanowire is specified by how far away the two intermediate control points $\left(P_{1}\right.$ and $\left.P_{2}\right)$ of the Bézier curve may lie, in the tangential sense, from a straight path connecting the two ends of the curve $\left(P_{0}\right.$ and $\left.P_{3}\right)$, as illustrated in Fig. 1(a). The nanowire itself, however, does not actually pass through $\mathrm{P}_{1}$ and $\mathrm{P}_{2}$.

In order to quantify the degree of curviness in the network, we define the curviness angle $\square_{c}$ with respect to the straight line connecting $P_{0}$ and $P_{3}$ such that possible anchor points of $P_{1}$ and $P_{2}$ may lie on normal line segments extending from points $1 / 3$ and $2 / 3$ of the distance from $P_{0}$ to $P_{3}$, satisfying the properties $\tan \left(\theta_{c} / 2\right) \leq\left|\tan \left(\theta_{P_{1}}\right) / 3\right| \leq \tan \left(\theta_{c}\right)$ and $\tan \left(\theta_{c} / 2\right) \leq$ $\left|\tan \left(\theta_{P_{2}}\right) \times 2 / 3\right| \leq \tan \left(\theta_{c}\right)$, as shown in Fig. 1(a). Here, $\theta_{P 1}$ and $\theta_{P_{3}}$ denote the angles formed by $P_{1}$ and $P_{2}$, respectively, referenced to the line $P_{0}-P_{3}$. By this definition, $\square_{c}=0^{0}$ corresponds to a perfectly straight nanowire network with no curviness, and the degree of curviness increases as $\square_{c}$ increases.

Once the curviness angle for the network is defined, $P_{1}$ and $P_{2}$ points of each nanowire is generated randomly within the allowed range specified above. As a result, the network contains nanowires with a statistical distribution of the degree of curviness generated within the constraints defined by the curviness angle. In the implementation of the simulation code, once the Bézier curves are generated, they are approximated by 8 straight line sub-segments. This number of segments was found, for high curviness, to be the smallest number such that using one more segment would not change the calculated network resistance by more than $1 \%$.

Furthermore, the length between the start and end points $\left(P_{0}\right.$ and $\left.P_{3}\right)$ is scaled to make the curved length of each nanowire equal to the nanowire length $l_{W}$ specified in the code.

In addition to curviness, to quantify the degree of nanowire alignment in the network, we define the alignment angle $\square_{a}$. The straight paths connecting $P_{0}$ and $P_{3}$ for each nanowire in the simulation are generated at random angles $\square$ with respect to the horizontal axis in the range $-\theta_{a} \leq \theta \leq \theta_{a}$ and $180-\theta_{a} \leq \theta \leq 180+\theta_{a}$, where $\square_{a}$ is the alignment angle defined in the range $0 \leq \theta_{a} \leq 90^{\circ}$, as shown in our previous work (Behnam et al., 2007, Hicks et al., 2009, Behnam et al., 2008). By this definition, $\square_{a}=90^{\circ}$ yields completely randomly distributed nanowires, whereas $\square_{a}=0^{\circ}$ yields nanowires perfectly aligned along the horizontal axis between Electrode 1 and Electrode 2. Figures 2(a)-(d) show four different nanowire networks that we have generated using the simulation code with different values of $\square_{c}$ and $\square_{a}$. 


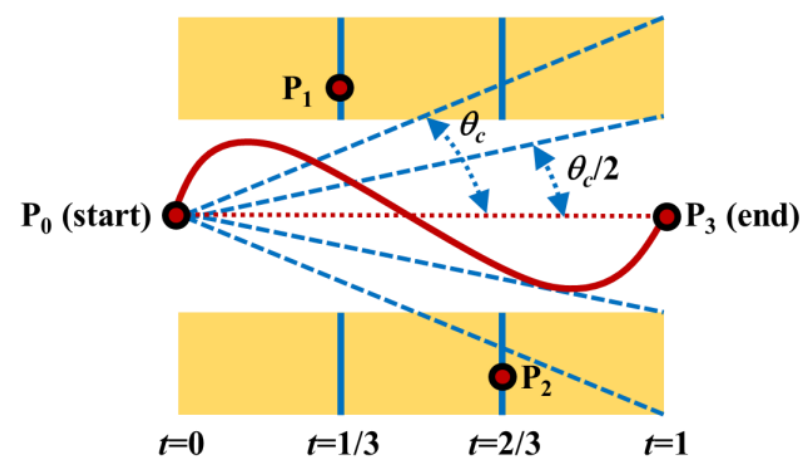

Figure 1. (a) Illustration of the generation of a curvy nanowire. $P_{0}, P_{1}, P_{2}$, and $P_{3}$ are the four control points of the cubic Bézier curve used to generate each curved nanowire. $P_{1}$ and $P_{2}$ lie $1 / 3$ and $2 / 3$ of the distance along the straight line joining P0 (start) and P3 (end) points. The curviness angle $\square_{c}$ is defined with respect to the straight line connecting $P_{0}$ and $P_{3}$ such that possible anchor points of $P_{1}$ and $P_{2}$ are limited to the vertical solid blue lines depicted in the figure. The red dotted line connecting $\mathrm{P}_{0}$ and $\mathrm{P}_{3}$ shows the effective straight length $l_{\text {eff }}$ and the red solid line is the nanowire length $l_{W}$.
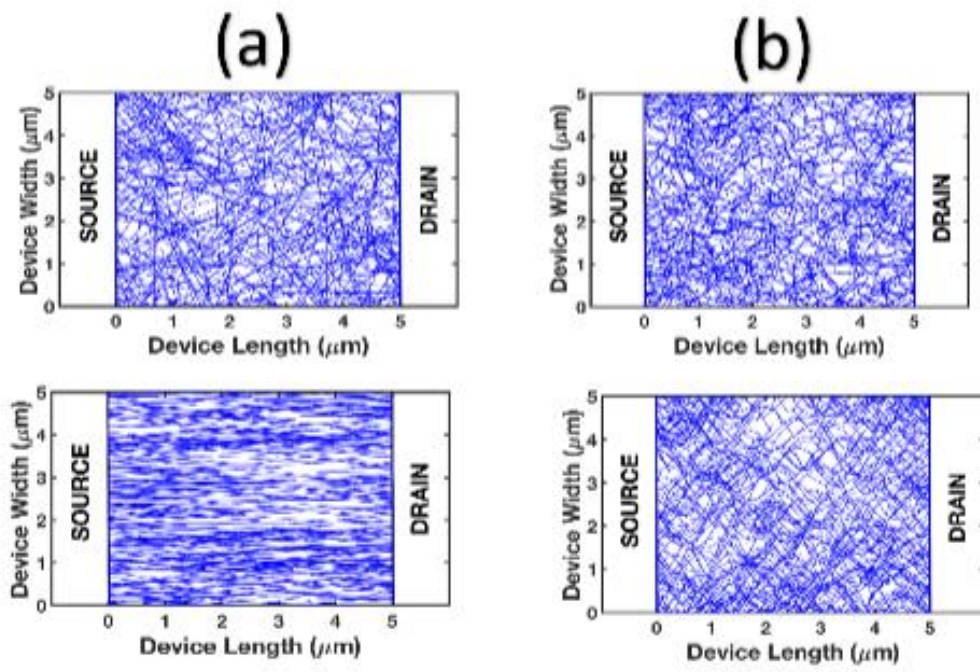

(c)

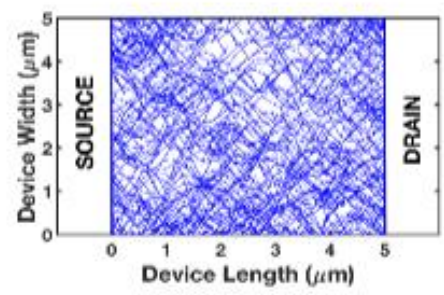

(d)

Figure 2. Nanowire networks generated using our Monte Carlo simulation code showing (a) randomly oriented $\left(\theta_{a}=90^{\circ}\right)$ straight nanowires $\left(\theta_{c}=0^{\circ}\right)$, (b) randomly oriented $\left(\theta_{a}=90^{\circ}\right)$ curvy nanowires $\left(\theta_{c}=81^{\circ}\right)$, (c) aligned $\left(\theta_{a}=\right.$ $\left.10^{\circ}\right)$ straight nanowires $\left(\theta_{c}=0^{\circ}\right)$, and $(\mathrm{d})$ aligned $\left(\theta_{a}=10^{\circ}\right)$ curvy nanowires $\left(\theta_{c}=81^{\circ}\right)$.

\section{Calculation of Resistivity}


There are two contributions to the resistivity of the network. The first is the nanowire-nanowire junction resistance, which is modeled as a fixed resistance $R_{J}$, and the second is the resistivity of the nanowire itself, which is modeled by $R_{l}$, the resistance of a nanowire having a length of $1 \mu \mathrm{m}$. As a result, the resistance $R_{d}$ of a nanowire segment of length $d$ microns between two junctions is given by $R_{d}=R_{l} \times d$. Previous experimental and theoretical work on metal nanowires have shown that the junction resistance dominates the overall resistivity of the network (Jagota and Tansu, 2015; Mutiso and Winey, 2013; Mutiso et al., n.d.); as a result, we have used $R_{J}=1000 R_{l}$ in the simulations. Junctions between nanowires, as well as nanowires and the two electrodes that occupy fixed locations in space, are identified and compiled into an adjacency matrix of resistors. Using the boundary conditions imposed by the electrode voltages, the overall network resistance is calculated using Modified Nodal Analysis (MNA) (Ho, 1975) and summing the currents at one electrode, as explained in detail previously (Behnam and Ural, 2007; Behnam et al., 2007; Hicks et al., 2009; Behnam et al., 2008; Hicks et al., 2009). In the plots presented in the next section, each resistivity data point represents the average of 400 different simulations of the network.

\section{Results and Discussion}

\section{Resistance Ratio and Nanowire Densities}

In most Monte Carlo simulations, it is assumed that junction resistance $\left(\mathrm{R}_{\mathrm{J}}\right)$ dominates nanowire resistance $\left(\mathrm{R}_{\mathrm{w}}\right)$. However, recent experiments have shown that, for metal nanowire networks such as Ag, junction resistance can be significantly lowered by post-deposition treatments such as thermal annealing, plasmonic welding, joule heating, and mechanical pressing. By tuning the junction-to-nanowire resistance ratio $\left(\mathrm{R}_{\mathrm{J}} / \mathrm{R}_{\mathrm{w}}\right)$, the resistivity of the network can be changed from a junction-dominated high resistance state (HRS) to nanowiredominated low resistance state (LRS). Figure 3 shows the log-log plot of normalized resistivity versus resistance ratio for five different nanowire densities ranging from $5 \mu \mathrm{m}^{-2}$ to $35 \mu^{-2}$. It can be seen from Figure 3 that, as the nanowire density decreases, the resistivity of the network increases. At fixed nanowire density, n, resistivity is initially constant for nanowire-dominated networks $\left(\mathrm{R}_{\mathrm{J}} / \mathrm{R}_{\mathrm{w}}<1\right)$. The ratio of resistivity for junction-dominated high resistance state (HRS) $\left(\mathrm{R}_{\mathrm{J}} / \mathrm{R}_{\mathrm{w}}=10^{3}\right)$ to nanowire-dominated low resistance state $(\mathrm{LRS})\left(\mathrm{R}_{\mathrm{J}} / \mathrm{R}_{\mathrm{w}}=10^{-3}\right)$ increases as 
nanowire density decreases. Decreasing nanowire density drives the network towards the percolation threshold, which magnifies the effect of junction resistance on resistivity.

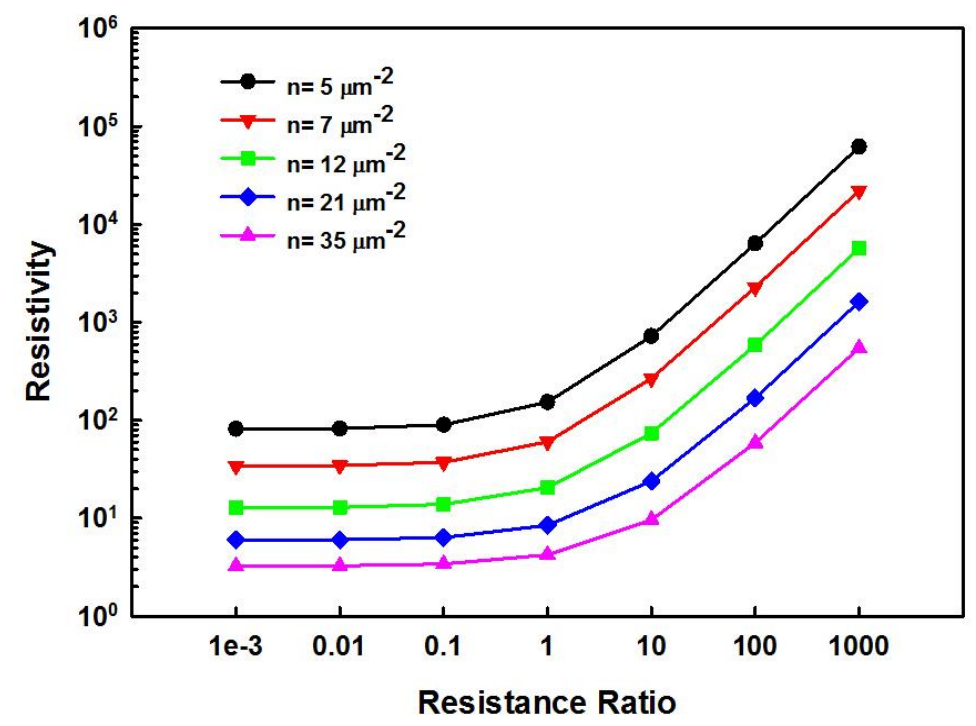

Figure 3. Log-log plot of normalized resistivity versus resistance ratio for five different nanowire densities ranging from $5 \mu \mathrm{m}^{-2}$ to $35 \mu \mathrm{m}^{-2}$, as labeled by different symbols. The other simulation parameters are nanowire length $l_{W}=2 \mu \mathrm{m}$, device length $L=10 \mu \mathrm{m}$, device width $W=10 \mu \mathrm{m}$, and alignment angle $\theta_{a}=90^{\circ}$ (i.e. randomly distributed). The solid line is the linear best-fit to the data.

\section{Resistance Ratio and Nanowire Length}

Figure 4 shows the log-log plot of the normalized resistivity versus resistance ratio for five different nanowire lengths $\left(1_{\mathrm{w}}\right)$ ranging from $1.3 \mu \mathrm{m}$ to $4 \mu \mathrm{m}$. It can be seen from Figure 4 that as nanowire length decreases, the resistivity of the network increases. At fixed nanowire density $l_{\mathrm{w}}$, resistivity is initially constant for nanowire-dominated networks, but increases as the network becomes junction-dominated. HRS to LRS resistivity ratio increases as $1_{\mathrm{w}}$ decreases. Decreasing $1_{\mathrm{w}}$ drives the network towards the percolation threshold, which magnifies the effect of junction resistance on resistivity. 


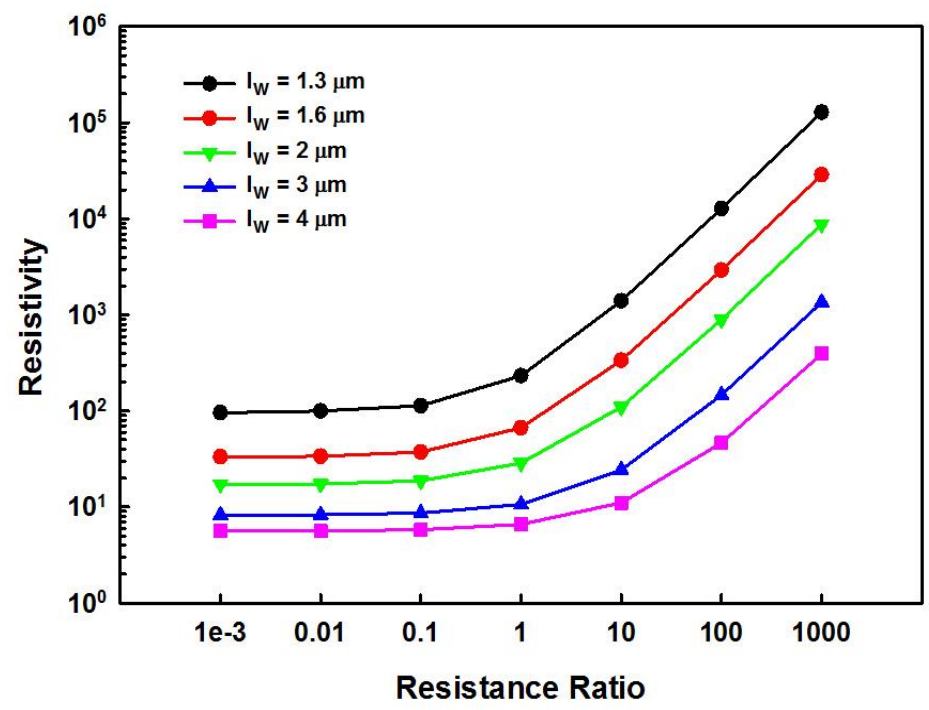

Figure 4. Log-log plot of normalized resistivity versus resistance ratio for five different nanowire lengths ranging from $1.3 \mu \mathrm{m}$ to $4 \mu \mathrm{m}$, as labeled by different symbols. The other simulation parameters are $\mathrm{n}=10 \mu \mathrm{m}^{-2}, \mathrm{~L}=$ $10 \mu \mathrm{m}, \mathrm{W}=10 \mu \mathrm{m}$, and $\theta \mathrm{a}=90^{\circ}$ (i.e. randomly distributed). The solid line is the linear best-fit to the data.

\section{Resistance Ratio and Curviness Angles}

Figure 5 shows the log-log plot of the normalized resistivity versus resistance ratio for five different curviness angles $\left(\theta_{c}\right)$ ranging from $0^{\circ}$ to $81^{\circ}$. It can be seen from Figure 5 that as the nanowire curviness angle increases, so does the resistivity of the network. At a fixed curviness angle $\theta_{c}$, resistivity is initially constant for nanowire-dominated networks, but increases as the network becomes junction-dominated. HRS to LRS resistivity ratio increases as the curviness angle increases. Increasing the curviness angle decreases the number of conduction paths and hence the connectivity of the network, which magnifies the effect of junction resistance on resistivity. 


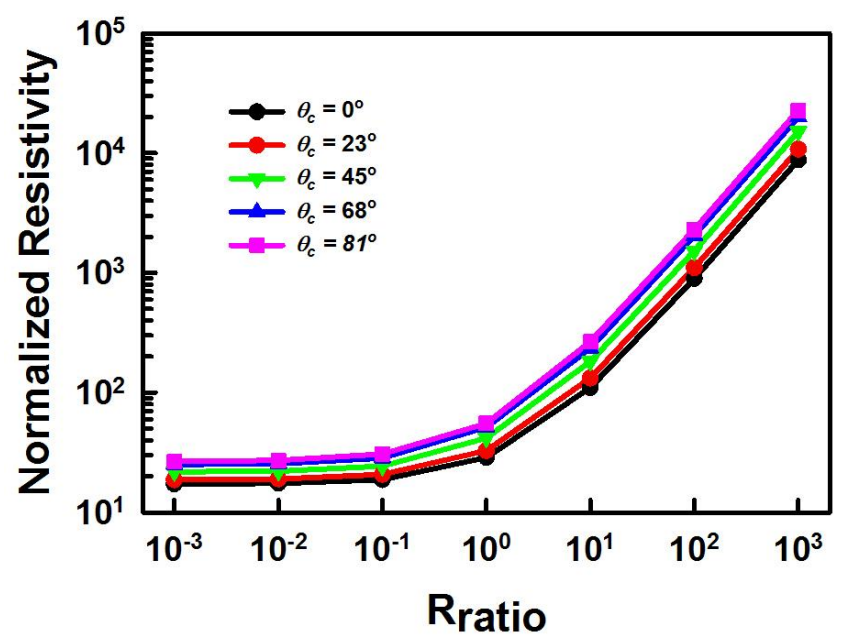

Figure 5. Log-log plot of normalized resistivity versus resistance ratio for five different curviness angles ranging from $0^{\circ}$ to $81^{\circ}$, as labeled by different symbols. The other simulation parameters are $\mathrm{n}=10 \mu \mathrm{m}^{-2}, \mathrm{~L}=10 \mu \mathrm{m}, \mathrm{W}=$ $10 \mu \mathrm{m}$, and $\theta \mathrm{a}=90^{\circ}$ (i.e. randomly distributed). The solid line is the linear best-fit to the data.

\section{Conclusions}

In summary, we have used Monte Carlo simulations to study the effect of junction resistance on the percolation resistivity of metal nanowire networks. We generated the curvy nanowires as 1D width less "sticks" using 3rd-order Bézier curves. The degree of curviness of the nanowires was determined by the curviness angle, which puts a geometrical constraint on the randomly generated locations of the two intermediate control points of the Bézier curve. Five vertically stacked 2D layers were used to form the nanowire network. The network resistivity was calculated assuming a resistance ratio junction resistance-dominated network over nanowire resistance-dominated, consistent with previous literature.

Using these simulations, we systematically studied the interaction between the effect of curviness and three other nanowire/device parameters on the network resistivity, namely nanowire density, nanowire length, and curviness angle in the network. In each case, we observed the change of the network resistivity from a junction-dominated high resistance state to a nanowire-dominated low resistance state. When evaluating network resistivity at different nanowire densities, we found that the ratio of resistivity for junction-dominated high resistance state to nanowire-dominated low resistance state increases as density increases. When we evaluated network resistivity at different nanowire lengths, we found that the ratio of resistivity for junction-dominated high resistance state to nanowire-dominated low resistance state increases as length decreases. When we evaluated network resistivity at different curviness 
angles, we found that the ratio of resistivity for junction-dominated high resistance state to nanowire-dominated low resistance state increases as curviness increases.

Our results show that nanowire density and length provide another internal knob to tailor the electronic properties of transparent, conductive metal nanowire networks without changing the volume of the nanowire material used. Furthermore, these results are not limited to metal nanowire networks, but can be extended to any 2D or quasi-2D network, film, or nanocomposite consisting of 1D nanoelements such as carbon nanotubes, graphene nanoribbons, nanowires, and nanorods of different materials. Computational studies, such as presented in this work, are an essential predictive tool for both developing a fundamental understanding of percolation transport and optimizing the electronic properties of transparent, conductive nanowire networks for a wide range of applications.

\section{Acknowledgements}

The authors would like to thank S. Mishra, J. Li, T.-Y. Tsai, and J. Hicks for help with simulations.

\section{References}

Ackermann, T., Neuhaus, R., \& Roth, S. (2016). The effect of rod orientation on electrical anisotropy in silver nanowire networks for ultra-transparent electrodes. Scientific Reports, 6. Retrieved from https://www.nature.com/articles/srep34289

Aurang, P., Doganay, D., Bek, A., Turan, R., \& Unalan, H. E. (2016). Silver nanowire networks as transparent top electrodes for silicon solar cells. Solar Energy, 141, 110-117. Retrieved from https://www.sciencedirect.com/science/article/pii/S0038092X16305539?via=ihub

Azuma, K., Sakajiri, K., Matsumoto, H., Kang, S., Watanabe, J., \& Tokita, M. (2013). Facile fabrication of transparent and conductive nanowire networks by wet chemical etching with an electrospun nanofiber mask template. Materials Letters, 115, 187-189. Retrieved from https://www.sciencedirect.com/science/article/pii/S0167577X13014420?via=ihub

Bao, W. S., Meguid, S. A., Zhu, Z. H., Pan, Y., \& Weng, G. J. (2013). Effect of carbon nanotube geometry upon tunneling assisted electrical network in nanocomposites. Journal of Applied Physics, 113. Retrieved from https://aip.scitation.org/doi/10.1063/1.4809767

Behnam, A., \& Ural, A. (2007). Computational study of geometry-dependent resistivity scaling in singlewalled carbon nanotube films. American Physical Society, 75(12). Retrieved from https://journals.aps.org/prb/abstract/10.1103/PhysRevB.75.125432 
Behnam, A., Guo, J., \& Ural, A. (2007). Effects of nanotube alignment and measurement direction on percolation resistivity in single-walled carbon nanotube films. Journal of Applied Physics, 102(4). Retrieved from https://aip.scitation.org/doi/10.1063/1.2769953

Behnam, A., Bosman, G., \& Ural, A. (2008). Percolation scaling of \$1/f\$ noise in single-walled carbon nanotube films. American Physical Society, 78(8). Retrieved from https://journals.aps.org/prb/abstract/10.1103/PhysRevB.78.085431

Berhan, L., \& Sastry, A. M. (2007). Modeling percolation in high-aspect-ratio fiber systems. II. The effect of waviness on the percolation onset. American Physical Society, 75(4). Retrieved from https://journals.aps.org/pre/abstract/10.1103/PhysRevE.75.041121

Borchert, J. W., Stewart, I. E., Ye, S., Rathmell, A. R., Wiley, B. J., \& Winey, K. I. (2015). Effects of length dispersity and film fabrication on the sheet resistance of copper nanowire transparent conductors. Royal Society of Chemistry, (34). Retrieved from https://pubs.rsc.org/en/Content/ArticleLanding/2015/NR/C5NR03671B\#!divAbstract

Chunyu Li, T. C. (2007). Continuum percolation of nanocomposites with fillers of arbitrary shapes. Applied Physics Letters, 90(17). Retrieved from https://aip.scitation.org/doi/10.1063/1.2732201

Coleman, J. N., \& De, S. (2011). The effects of percolation in nanostructured transparent conductors | MRS Bulletin. MRSBulletin, 36(10), 774-781. Retrieved from https://www.cambridge.org/core/journals/mrs-bulletin/article/effects-of-percolation-innanostructured-transparent-conductors/A7D475FB94E4EC9421C4A2B3A6D84138

Dalmas, F., Dendievel, R., Chazeau, L., Cavaille, J., \& Gauthier, C. (2006). Carbon nanotube-filled polymer composites. Numerical simulation of electrical conductivity in three-dimensional entangled fibrous networks. Acta Materialia, 54(11), 2923-2931. Retrieved from https://www.sciencedirect.com/science/article/pii/S1359645406001662?via=ihub

H. M. Ma, X., Gao, \& Tolle, T. B. (2010). Monte Carlo modeling of the fiber curliness effect on percolation of conductive composites. Applied Physics Letters, 96(6). Retrieved from https://aip.scitation.org/doi/10.1063/1.3309590

Hecht, D. S., Hu, L., \& Irvin, G. (2011). Emerging Transparent Electrodes Based on Thin Films of Carbon Nanotubes, Graphene, and Metallic Nanostructures. Advanced Materials. Retrieved from https://onlinelibrary.wiley.com/doi/full/10.1002/adma.201003188

Hicks, J., Behnam, A., \& Ural, A. (2009). Resistivity in percolation networks of one-dimensional elements with a length distribution. American Physical Society, 79(1). Retrieved from https://journals.aps.org/pre/abstract/10.1103/PhysRevE.79.012102

Hicks, J., Behnam, A., \& Ural, A. (2009). A computational study of tunneling-percolation electrical transport in graphene-based nanocomposites. Applied Physics Letters, 95(21). Retrieved from https://aip.scitation.org/doi/10.1063/1.3267079

Hu, L., Kim, H. S., Li, J. Y., Peumans, P., \& Cui, Y. (2010). Scalable Coating and Properties of Transparent, Flexible, Silver Nanowire Electrodes. ACS Nano. Retrieved from https://pubs.acs.org/doi/10.1021/nn1005232 
Hu, L., Wu, H., \& Cui, Y. (2011). Metal nanogrids, nanowires, and nanofibers for transparent electrodes | MRS Bulletin. MRSBulletin, 36(10), 760-765. Retrieved from https://www.cambridge.org/core/journals/mrs-bulletin/article/metal-nanogrids-nanowires-andnanofibers-for-transparent-electrodes/8EF4EC1BA0FD23227AE5BB743FBDD89F

Jagota, M., \& Tansu, N. (2015). Conductivity of Nanowire Arrays under Random and Ordered Orientation Configurations. Scientific Reports, 5. Retrieved from https://www.nature.com/articles/srep10219

Kim, B. S., Sin, K., Pyo, J. B., Lee, J., Son, J. G., Lee, S., \& Park, J. H. (2016.). Reversibly Stretchable, Optically Transparent Radio-Frequency Antennas Based on Wavy Ag Nanowire Networks. ACS Applied Materials \& Interfaces. Retrieved from https://pubs.acs.org/doi/10.1021/acsami.5b10317

Langley, D., Giusti, G., Mayousse, C., Celle, C., Bellet, D., \& Simonato, J. (2013). Flexible transparent conductive materials based on silver nanowire networks: A review. Nanotechnology, 24(45). Retrieved from https://iopscience.iop.org/article/10.1088/0957-4484/24/45/452001/meta

Li, C., Thostenson, E. T., \& Chou, T. (2007). Effect of nanotube waviness on the electrical conductivity of carbon nanotube-based composites. Composites Science and Technology, 68(6). Retrieved from https://www.sciencedirect.com/science/article/pii/S0266353807004502?via=ihub

Mutiso, R. M., Sherrott, M. C., Rathmell, A. R., Wiley, B. J., \& Winey, K. I. (2013). Integrating Simulations and Experiments To Predict Sheet Resistance and Optical Transmittance in Nanowire Films for Transparent Conductors. ACS Nano. Retrieved from https://pubs.acs.org/doi/10.1021/nn403324t

Mutiso, R. M., \& Winey, K. I. (2013). Electrical percolation in quasi-two-dimensional metal nanowire networks for transparent conductors. American Physical Society, 88(3). Retrieved from https://journals.aps.org/pre/abstract/10.1103/PhysRevE.88.032134

Simoneau, L., Villeneuve, J., \& Rochefort, A. (2015). Electron percolation in realistic models of carbon nanotube networks. Journal of Applied Physics, 118(12). Retrieved from https://aip.scitation.org/doi/10.1063/1.4931676

Stauffer, D., \& Aharony, A. (1985). Introduction to Percolation Theory. Taylor and Francis. doi:10.4324/9780203211595

Takeda, T., Shindo, Y., Kuronuma, Y., \& Narita, F. (2011). Modeling and characterization of the electrical conductivity of carbon nanotube-based polymer composites. American Physical Society, 79(2). Retrieved from https://www.sciencedirect.com/science/article/pii/S0032386111005350?via=ihub

White, S. I., DiDonna, B. A., Mu, M., Lubensky, T. C., \& Winey, K. I. (2009). Simulations and electrical conductivity of percolated networks of finite rods with various degrees of axial alignment. American Physical Society, 79(2). Retrieved from https://journals.aps.org/prb/abstract/10.1103/PhysRevB.79.024301

Ye, S., Rathmell, A. R., Ian E. Stewart, Y. H., Wilson, A. R., Chen, Z., \& Wiley, B. J. (2013). A rapid synthesis of high aspect ratio copper nanowires for high-performance transparent conducting films. Royal Society of Chemistry, 20. Retrieved from https://pubs.rsc.org/en/Content/ArticleLanding/2014/CC/C3CC48561G\#!divAbstract 
Ye, S., Rathmell, A. R., Chen, Z., Stewart, I. E., \& Wiley, B. J. (2014). Metal Nanowire Networks: The Next Generation of Transparent Conductors. Advanced Materials. Retrieved from https://onlinelibrary.wiley.com/doi/full/10.1002/adma.201402710

Yi, Y. B., Berhan, L., \& Sastry, A. M. (2004). Statistical geometry of random fibrous networks, revisited: Waviness, dimensionality, and percolation. Journal of Applied Physics, 96(3). Retrieved from https://aip.scitation.org/doi/10.1063/1.1763240

Yu, Y., Song, S., Bu, Z., Gu, X., \& Sun, L. (2013). Influence of filler waviness and aspect ratio on the percolation threshold of carbon nanomaterials reinforced polymer nanocomposites. Journal of Material Science, 48(17), 5727-5732. Retrieved from https://link.springer.com/article/10.1007/s10853-013-7364-z 